Post ural stabi I ity during one- leg stance on an unst abl e moving $\mathrm{pl}$ at formand i ts rel at i onshi $p$ with each I eg

\begin{tabular}{|l|l|}
\hline 著者 & $\begin{array}{l}\text { Noguchi Takanor i , Demur a Shi ni chi , Nakagawa } \\
\text { Toshi har u }\end{array}$ \\
\hline $\begin{array}{l}\text { j our nal or } \\
\text { publ i cat i on ti t l e }\end{array}$ & Percept ual and Not or Ski I I s \\
\hline vol une & 116 \\
\hline number & 2 \\
\hline page r ange & $555-563$ \\
\hline year & $2013-08-01$ \\
\hline URL & ht t p: //hdl . handl e. net /2297/35634 \\
\hline
\end{tabular}




\title{
POSTURAL STABILITY DURING ONE-LEG STANCE ON \\ AN UNSTABLE MOVING PLATFORM AND ITS
}

RELATIONSHIP WITH EACH LEG

\author{
TAKANORI NOGUCHI \\ Fukui University of Technology \\ SHIN-ICHI DEMURA \\ Graduate School of Natural Science \& Technology, Kanazawa University \\ TOMOHARU NAKAGAWA \\ NTT WEST Corporation
}

Author's Note

Please address all correspondence to: Takanori Noguchi,

Fukui University of Technology,

Gakuen 3-6-1, Fukui, Fukui 910-8505, JAPAN

Tel: +81- 776-29-2620

E-mail: t-noguchi@fukui-ut.ac.jp 
Keywords: manipulation leg, nonmanipulation leg, postural stability, one-leg stance

Summary: This study examined the laterality of the supporting leg in postural stability during a one-leg stance test on an unstable moving platform and its practice effect. Thirty male university students were evaluated using the omnidirection stability index (OSI) during a one-leg stance test on an unstable moving platform (three trials). The leg used when kicking a ball was defined as the manipulation leg (ML) and the supporting leg was defined as the nonmanipulation leg (NML). According to the two-way repeated measures ANOVA (trials $\times$ legs), a significant difference was observed only in the leg factor, and OSI values of the second and third trials were less in ML than in NML. The correlation between both legs was significant but not high. ML was superior to NML with regard to stability, and practice effect with trials was not evident in either leg. 
Because one arm is preferentially used when performing movements that require dexterity of the upper limbs, a dominant hand develops (Demura, Sato, \& Nagasawa, 2009; Noguchi, Demura, Nagasawa, \& Uchiyama, 2005). In contrast, unlike the upper limbs, both legs are generally used in almost the same way as most of the basic motions in daily life such as standing, walking, and running require touching the ground with both legs simultaneously or alternately, or are achieved by supporting the body with one or both legs; therefore, there is little opportunity to recognize the dominant leg. In addition, because these movements are already automated (“routinized”) and the same motions are repeated, particularly without conscious knowledge regarding the same (Kurt, 1981), we lack awareness of the dominant leg; however, there are tasks that demonstrate laterality of the manipulating leg. For example, we generally use the leg that is easier to manipulate when kicking a soccer ball.

This phenomenon occurs if subjects concentrate on a complex motion and use the preferred leg because the given motion is very difficult and hence cannot be automated. During such a task, the nonpreferred leg supports the body and has a role in balancing the body to accurately kick the ball (Matsuda, Demura, \& Uchiyama, 2008). In this case, because the task of supporting the body is easier than that of controlling the ball, we are not particularly attentive toward the movements of the supporting leg. 
However, there are also situations when the supporting leg is forced to perform a more

difficult task (Matsuda, Demura, \& Nagasawa, 2011: Paillard, Noé, Rivière, Marion, Montoya, \& Dupui, 2006) such as maintenance of postural stability during a one-leg stance test. It has been reported that because of the low complexity level, simple one-leg support does not depict a clear difference between the manipulation leg (ML) and the nonmanipulation (NML; supporting) leg (Matsuda, Demura, \& Demura, 2010). However, standing on an unstable, moving platform has also been used for rehabilitation of disorders and for balance training of athletes (Demura. 2011) because such a task is more difficult than keeping the posture stable on a flat or fixed platform (Gstöttner, Neher, Scholtz, Millonig, Lembert, \& Raschner, 2009). Standing on one leg on an unstable moving platform is extremely difficult for the supporting leg. Therefore, lateral dominance in the legs may appear in a similar manner when kicking a ball. However, conclusions on the abovementioned lateral dominance have not been consistent even in recent studies (Gstöttner, et al., 2009: Golomer \& Mbongo, 2004).

Several tests can be used to evaluate the nerve functions of upper limbs. These tests have revealed that these functions generally improve performances with each trial: in short, the practice effect is observed (Noguchi, et al., 2005). This is considered to occur because test tasks are generally very complicated and unfamiliar and require the exertion of dexterity and skill of hands, which are not usually experienced in daily life. In addition, with regard to the 
lower limbs, because movements such as kicking a ball are performed frequently for physically active young people, a test using these exercise tasks indicates little practice effect. The one-leg stance on an unstable supporting base used in the present study may demonstrate the practice effect because the task is unfamiliar and complicated. Moreover, because the practice effect may affect the interpretation of measurement results, sufficient consideration is required. In addition, it does not necessarily present the same tendency in both legs. In short, when dominance exists in legs, the dominant leg may become accustomed to tasks quicker compared with the nondominant leg. Shigeshima, Fujiwara, Ogoma, Ohkura, and Nakaya (2009) reported that the stride length, duration of one-leg stance, and one-swing duration of both legs in gait motion revealed high correlations (>0.8). During running and standing-up movements, the relationship between both legs is very strong (Kurt, 1981). We believe that this relationship develops to ensure stable motion by using both legs equally. However, in the case of one-leg stance on an unstable moving platform, because each leg has an important role in maintaining postural stability, the relationship between the legs may differ from that during gait motion. This study will be valuable for clarifying the laterality of both legs, which is one of the important factors affecting performances in various sports. In addition, in rehabilitation programs, knowledge regarding the original difference between both legs is important to 
understand the normal state appropriately when the injured lower limbs recover (Kovaleski,

Heitman, Gurchiek, Erdmann, \& Trundle, 1997).

The purpose of the present study was to examine the laterality of the supporting leg in postural stability when standing on one leg on an unstable moving platform and to examine its practice effects.

\section{Methods}

\section{Subjects}

The subjects in the present study were 30 male university students (age, $20.3 \pm 1.0$ years;

height, $172.2 \pm 6.2 \mathrm{~cm}$; weight, $66.7 \pm 8.2 \mathrm{~kg})$. All subjects were healthy young adults without any disability or injury (e.g., sprained ankle in either leg), who belonged to athletic clubs for $>6$ years. Before the measurements were recorded, the purpose and procedure were explained in detail, and informed consent was obtained from all participants. This experimental protocol was approved by the Ethics Committee on Human Experimentation of the Faculty of Human Science, Kanazawa University (Number. 2012-03: Kanazawa, Japan).

Matsuda, et al. (2010) categorized legs into a kicking leg and a supporting leg when kicking a ball. In this study, the former leg was considered ML and the latter leg as NML. 


\section{Device and evaluation variables}

Dyjoc Board Plus (SAKAI med, SV-200) was used to estimate stability during the one-leg stance on an unstable platform. This measurement device (in which the bottom of a ship-shaped boss is attached to the central part of the back of a flat board) can slant up to $12^{\circ}$ backward and forward and $7^{\circ}$ laterally (Fig.1). This device simultaneously measures the anteroposterior and right-left inclination angles relative to the horizontal plane using an in-built accelerometer with a sampling rate of $40 \mathrm{~Hz}$. Data are sent directly to a personal computer for analyses using Dyjoc Board Plus software (SAKAI med, SV-200). Acceleration changes of the board, which was used to measure the variations in subject's balance, were calculated using angle variations (values without units) as X-axis (back and forth) and Y-axis (right and left; Fig. 2).

*** Insert Fig 1 here

*** Insert Fig 2 here ***

The omnidirection stability index (OSI), which reflects postural stability, was calculated using the angle data from the gradient of anteroposterior and right-left directions measured 
using the abovementioned device. This index was used as a parameter to evaluate stability during the one-leg stance test. Moreover, the following formula was used to calculate OSI:

$$
=\sqrt{\frac{\sum_{i=1}^{n}\left(0-X_{i}\right)^{2}+\sum_{i=1}^{n}\left(0-Y_{i}\right)^{2}}{n}} \text { OSI }
$$

n: number of sampling data

Xi: sampling data of the $\mathrm{X}$-axis (back and forth)

Yi: sampling data of the Y-axis (right and left)

Therefore, in rehabilitation programs, knowledge regarding the original difference between both legs is important to understand the normal state appropriately when the injured lower limbs recover it is interpreted that the smaller the OSI, the more stable the upright posture.

\section{Measurement procedure}

First, all subjects were divided randomly into two groups and measurement was

performed with ML or NML. When the first measurement was performed with ML, the next one was performed with NML. The test protocol and measurement was performed as follows:

1) ML and NML were measured. 
2) The plantar surfaces of the supporting feet were placed on the landmarks with respect to the $\mathrm{X}$ - and Y-axes marked on the center of the board face (plain face), while placing the other foot on the ground.

3) After observing the target of the monitor before subjects, the subjects lifted the nonsupporting leg after receiving the tester's signal and continued to stand on one leg.

4) The nonsupporting leg did not touch the supporting leg during the one-leg stance test.

5) The measurement was recorded for 1 min after the 5-s one-leg stance (total $65 \mathrm{~s}$ ).

After one practice trial, the test was performed three times with each leg. A 1-min rest interval was taken between trials to eliminate the influence of previous trials and fatigue. If subjects lost postural balance and fell from the board during measurement, we performed the measurement again.

Three subjects fell from the board in one trial alone; measurements for these subjects were recorded again after a 1-min rest interval, and the respective values of each of the three trials were used for analyses.

At instances when the edge of the board touched the ground, the data were recognized as "at maximum inclination angle” ( $12^{\circ}$ backward and forward and $7^{\circ}$ laterally) and were sampled accordingly. 


\section{Statistical analyses}

Two-way repeated measures ANOVA was used to assess the differences between trial means $(3 \times 2$ : trials $\times$ legs). If a significant main effect or interaction was observed, a multiple comparison test was performed using Tukey's honestly significant difference (HSD) method. Size of the mean difference was assessed by the effect size (ES). In addition, intraclass correlation coefficients (ICCs) were calculated to evaluate the trial-to-trial reliability. The ICC $(1, k)$ type (average measure intraclass correlation) was used in this study, and IBM SPSS Statistics ver. 20.0 was used for analyses. The relationship between the variables of ML and NML were examined using Pearson's correlation coefficient test. The mean difference in OSI values of both legs was examined by a paired $t$-test. Statistical significance $(\alpha)$ was set at $p<$ 0.05 .

\section{Results}

Table 1 presents the results of the two-way ANOVA (trials and legs) and a multiple comparison test for OSI. The interaction observed was not statistically significant $[\mathrm{F}(2,58)=$ 1.52; $p>0.05]$. Moreover, significance in one leg factor alone (ML and NML) was observed [F $(1,29)=6.04 ; p<0.05]$. A multiple comparison test revealed that OSI values at the second and 
third trials were significantly lesser for ML than those for NML (Table 1). Effect sizes of OSI between ML and NML were 0.34 and 0.48 , respectively (Table 1 ).

\section{*** Insert Table 1 here ***}

ICCs of the three OSI trials in ML and NML were $0.81(\mathrm{~F}=3.10, p>0.05)$ and $0.89(\mathrm{~F}=$ $0.27, p>0.05)$.

The mean and standard deviation values of the three OSI trials were $2.02 \pm 0.40$ in ML and $2.32 \pm 0.72$ in NML. A correlation between both legs was significant but moderate $(r=0.48, p$ $<0.05)$. Furthermore, a significant difference was observed between the mean values of both legs $(\mathrm{t}=2.65, p<0.05)$, and ES was 0.44 .

\section{Discussion}

ML in the present study was decided on the basis of the definition set by Matsuda, et al. (2010): it was the leg that was used preferentially when kicking a ball. When controlling a ball, ML is the leg manipulating the ball, while the other leg is supporting the body. From these results, it was clarified that using ML as the supporting leg produces better postural stability on an unstable moving platform compared with that when using NML, which is the supporting leg 
when kicking a ball. To maintain postural stability, the body's center of gravity must be kept within a certain definite range of the support base (Matsuda, et al., 2010). In short, because of the moving support base, subjects must maintain stability under conditions in which the balance of the whole body is poor. Therefore, stimulation at the sole of the supporting leg and postural control by muscle responses around the ankle joint are required more than those in static one- or two-leg stances (Ikai, Tatsuno, \& Miyano, 2006).

ML is generally used for movements that cannot be performed without consciousness such as ball control. In contrast, NML plays an important part in support when kicking a ball. In this case, the role of NML in postural control is less important. However, in the case of a one-leg stance on an unstable platform, as used in the present study, the supporting leg is forced to not only merely support the body but also maintain postural stability on the unstable platform. From the present results, it was clarified that using ML as the supporting leg is better than using NML to maintain stable postural control. The results stated above suggest that ML is superior in supporting actions (i.e., in maintaining stable posture), and this superiority demonstrated the existence of laterality in the lower limbs.

Noguchi, et al. (2005) reported that the record improved with an increase in the number of trials in the Purdue Pegboard test and the Moving Beans with Tweezers test using the upper limbs. With regard to tests of the upper limbs (which relate closely to nerve function), a certain 
number of trials are necessary until the subjects can fully exert their primary ability. Thus, the measured values gradually improve with each trial because of the practice effect, and stable performances are observed after a certain number of trials (Noguchi, et al., 2005). The one-leg stance used in the present study requires exertion of leg strength on an unstable platform, and nerve function is thought to contribute to maintenance of postural stability more than the one-leg stance on stable ground. Therefore, it was assumed that the practice effect would be observed. However, no significant difference was observed among the trials in both legs, and high reliability was confirmed as well. The age of the subjects (young individuals) may have affected the results because they would have immediately comprehended the task as a result of their strong interest in the tasks along with their developed nerve function. It was clarified that in the case of young subjects, large variations in performances do not occur even if fewer trials are performed, and sufficiently reliable data can be obtained.

Although a significant correlation $(r=0.48)$ was observed between OSI of ML and NML, the value was not considerably high. Unlike the lower limbs, laterality of the upper limbs is remarkable (Noguchi, Demura, \& Aoki, 2009). A pursuit rotor task that required fine motor skills of the hands was reported to have a significant and moderate correlation between the dominant and nondominant hands ( $r=0.66$; Noguchi, Demura, Nagasawa, \& Uchiyama, 2009). 
In contrast, it was reported that relationships between stride $(r=0.97)$, stance phase time $(r=$ 0.94), or swing phase time ( $\mathrm{r}=0.80$ ) of both legs during walking (Shigeshima, et al., 2009) are very high. Both legs are equally used symmetrically while walking; therefore, correlations between the above parameters of both legs during walking would increase naturally. However, in the present study, ML and NML accomplished this task independently. Strictly speaking, both feet and legs are not anatomically and functionally symmetrical. Similar to the significant differences observed between their mean values, the results obtained using ML and NML are different even when performing the same task (Demura, Yamaji, Goshi, \& Nagasawa, 2001). Taken together, the above results suggest the presence of (not necessarily high) significant interaction.

Because stable standing posture using both legs is an unconscious supporting movement, we do not feel that one leg is dominant over the other. However, if maintaining stability requires concentration, laterality may also appear in the lower limbs. At this point, it will be necessary to clarify the characteristics of the dominant leg for other movements as well as to examine in detail the laterality of the lower limbs. In addition, the present study was based on data recorded in male subjects alone. Thus, because a gender difference is observed in functions related to lateral dominance or postural control, such as brain structure and muscle 
strength, it will be necessary to examine these characteristics for females as well in future studies.

\section{Conclusion}

Postural stability during one-leg stance on an unstable moving platform was observed to be superior in ML (ball-kicking leg) than in NML. In addition, the practice effect was not evident in either leg. Moreover, the relationship between one-leg stance stability of ML and NML was not considerably strong.

\section{References}

Demura, S. (2005) Textbook of Health and Sports Science. Tokyo: Kyorinsyoin. p. 107-124.

\section{[Japanese]}

Demura S. (2011) Health and a sports science lecture the 2nd edition. Tokyo: Kyorinsyoin.

\section{[Japanese ]}

Demura, S., \& Sato, S. (2010) Lower limb laterality characteristics based on the relationship between activities and individual laterality. Gazzetta Medica Italiana, 169(5), 181-191. 
Demura, S., Kitabayashi, T., Kimura, A., \& Matsuzawa, J. (2005) Body sway characteristics during a static upright posture in healthy and disordered elderly. Journal of Aging and Physical Activity, 24(5), 551-555.

Demura, S., Yamaji, S., Goshi, F., \& Nagasawa, Y. (2001) Lateral dominance of legs in maximal muscle power, muscular endurance, and grading ability. Perceptual and Motor Skills, 93(1), 11-23.

Golomer. E., \& Mbongo, F. (2004) Does footedness or hemispheric visual asymmetry influence center of pressure displacements? Neuroscience Letters, 367(2), 148-151.

Gstöttner, M., Neher, A., Scholtz, A., Millonig, M., Lembert, S., \& Raschner, C. (2009) Balance ability and muscle response of the preferred and nonpreferred leg in soccer players. Motor Control, 13(2), 218-231.

Ikai, T., Tatsuno, H., \& Miyano, S. (2006) Relationship between walking ability and balance function. The Japanese Association of Rehabilitation Medicine, 43, 828-833.

Kovaleski, J. E., Heitman, R. J., Gurchiek, L. R., Erdmann, J. W., Trundle, T. L. (1997) Reliability and Effects of Leg Dominance on Lower Extremity Isokinetic Force and Work Using the Closed Chain Rider System. Journal of Sport Rehabilitation, 6(4), 319-326. Kurt, M. (1981). Bewegungslehre. (Akira Kaneko, Trans.) Tokyo: Taisyukan. p.401-408. 
Matsuda, S., Demura, S., \& Demura, T. (2010) Examining differences between center of pressure sway in one-legged and two-legged stances for soccer players and typical adults. Perceptual and Motor Skills, 110(3), 751-760.

Matsuda, S., Demura, S., \& Nagasawa, Y. (2011) Static one-legged balance in soccer players during use of a lifted leg. Perceptual and Motor Skills, 111(1), 167-177.

Matsuda, S., Demura, S., \& Uchiyama, M. (2008) Centre of pressure sway characteristics during static one-legged stance of athletes from different sports. Journal of Sports Science, 26(7), 775-779.

Noguchi, T., Demura, S., \& Aoki, H. (2009) Superiority of the dominant and nondominant hands in static strength and controlled force exertion. Perceptual of Motor Skills, 109(2), 339-346.

Noguchi, T., Demura, S., Nagasawa, Y., \& Uchiyama, M. (2005) The practice effect and its difference of the pursuit rotor test with the dominant and non-dominant hands. Journal of Physiological Anthropology and Applied Human Science, 24(6), 589-593.

Noguchi, T., Demura, S., Nagasawa, Y., \& Uchiyama, M. (2009) Influence of measurement order by dominant and nondominant hands on performance of a pursuit-rotor task. Perceptual and Motor Skills, 108, 1-10. 
Paillard, T., Noé, F., Rivière, T., Marion, V., Montoya, R., Dupui, P. (2006) Postural performance and strategy in the unipedal stance of soccer players at different levels of competition. Journal of Athletic Training. 41(2), 172-176.

Shigeshima, K., Fujiwara, T., Ogoma, Y., Ohkura, M., \& Nakaya, H. (2009) Symmetry of step length and temporal variability in gait of people without impairment. The Journal of Japan Academy of Health Science, 12(1), 25-30.

Yamamoto, T. (2010) Why do you need measurement and evaluation? Training Journal, 32(1), 12-15.

Wakano, S., Takeda, T., Nakajima, K., Kurokawa, K., Ishigami, K. (2011) Effect of experimental horizontal mandibular deviation on dynamic balance. Journal of Prosthodontic Research, 55(4), 228-233. 

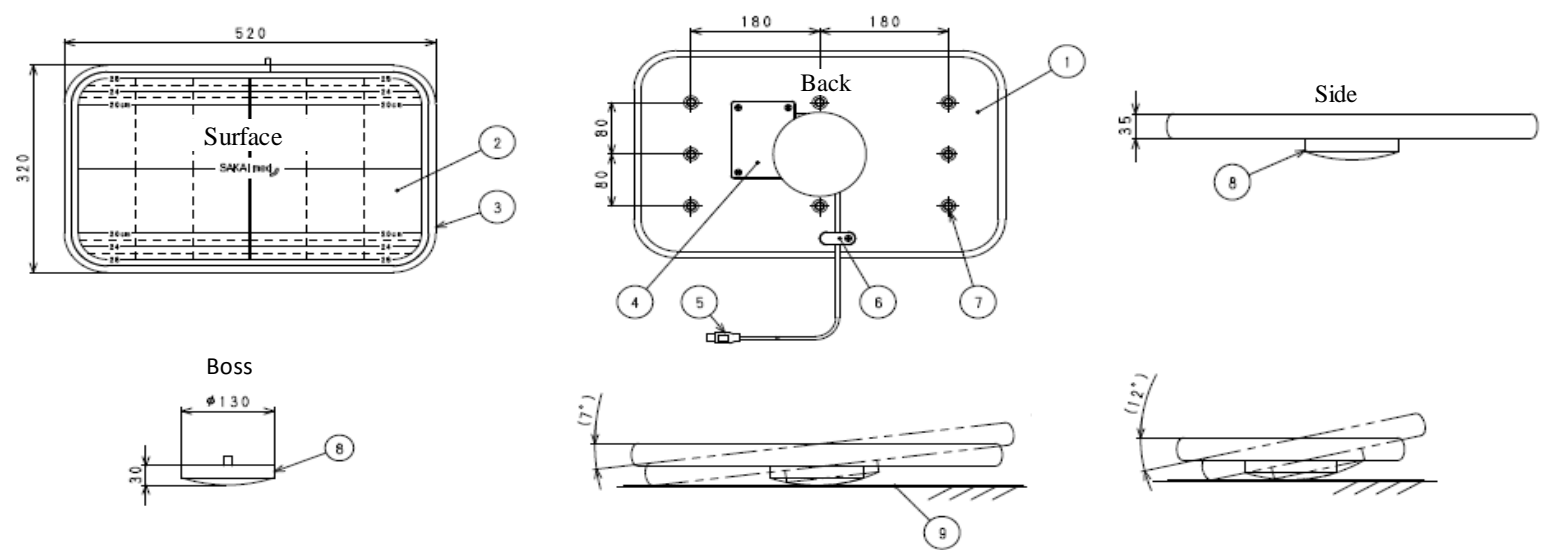

Fig 1. The measurement device (Dyjoc Board Plus)

Names of the parts

1. Main unit (length $(320 \mathrm{~mm}) \times$ width $(520 \mathrm{~mm}) \times$ height $(35 \mathrm{~mm})$

2. Foot position guide sheet

3. Soft edge

4. Control device

5. Universal serial bus (USB) cable

6. Barrier to prevent falling

7. Attachment position of boss

8. Boss (bottom type)

9. Rubber mat 

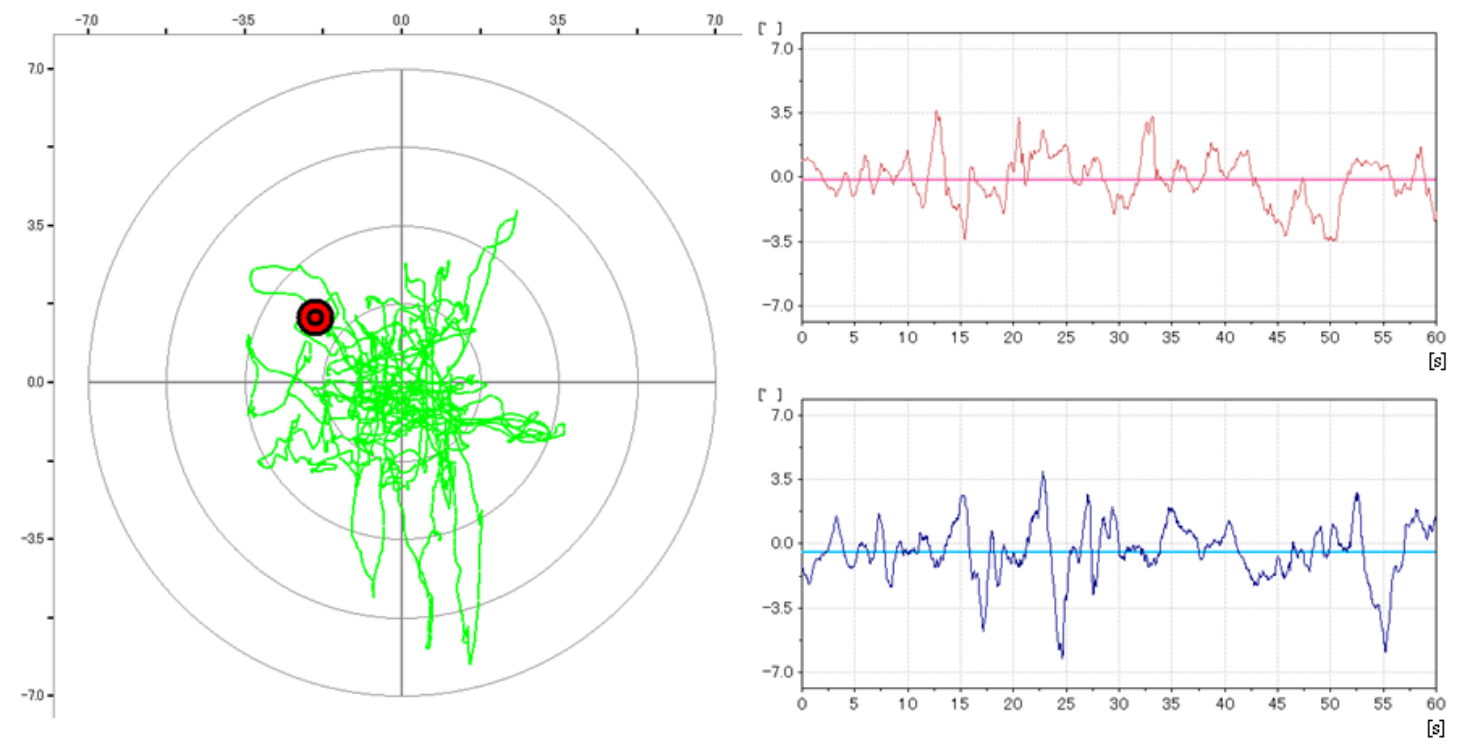

Fig 2. OSI sampling data 
Table 1. two-way ANOVA (trials $\times$ leg) of OSI

\begin{tabular}{|c|c|c|c|c|c|c|c|c|c|c|c|c|}
\hline \multirow[b]{2}{*}{$n=30$} & \multicolumn{3}{|c|}{$\mathrm{ML}$} & \multicolumn{3}{|c|}{ NML } & \multicolumn{3}{|c|}{ Difference } & \multirow{2}{*}{\multicolumn{2}{|c|}{ F-value }} & \multirow[b]{2}{*}{ Tukey's HSD } \\
\hline & $1 \mathrm{st}$. & 2nd & 3rd. & $1 \mathrm{st}$. & 2nd. & 3rd. & $1 \mathrm{st}$. & 2nd. & 3rd. & & & \\
\hline Mean & 2.17 & 2.07 & 1.99 & 2.35 & 2.29 & 2.34 & -0.18 & -0.22 & -0.36 & $\underline{F 1}$ & $6.04 *$ & 2nd \& 3rd: $M L<N M L$ \\
\hline SD & 0.48 & 0.49 & 0.40 & 0.59 & 0.74 & 0.75 & 0.66 & 0.62 & 0.66 & $\underline{\mathrm{F} 2}$ & $1.28 \mathrm{~ns}$ & \\
\hline Max & 3.20 & 2.94 & 2.62 & 4.00 & 4.35 & 4.43 & 0.88 & 0.88 & 0.42 & IN & $1.52 \mathrm{~ns}$ & effect size \\
\hline Min & 1.19 & 1.22 & 1.15 & 1.18 & 1.17 & 1.06 & -2.22 & -2.00 & -2.19 & & & 2nd. : $0.34,3$ rd. : 0.48 \\
\hline
\end{tabular}

F1: leg (ML : manipulation leg, NML : non-manipulation leg), F2 : trials, IN : interaction ns : no signifcant, $*: p<0.05$ 